The DARHT Scattering Wire Spectrometer: Operation and Checkout on ETA II

T. J. Fessenden

July 6, 2005 
This document was prepared as an account of work sponsored by an agency of the United States Government. Neither the United States Government nor the University of California nor any of their employees, makes any warranty, express or implied, or assumes any legal liability or responsibility for the accuracy, completeness, or usefulness of any information, apparatus, product, or process disclosed, or represents that its use would not infringe privately owned rights. Reference herein to any specific commercial product, process, or service by trade name, trademark, manufacturer, or otherwise, does not necessarily constitute or imply its endorsement, recommendation, or favoring by the United States Government or the University of California. The views and opinions of authors expressed herein do not necessarily state or reflect those of the United States Government or the University of California, and shall not be used for advertising or product endorsement purposes.

This work was performed under the auspices of the U.S. Department of Energy by University of California, Lawrence Livermore National Laboratory under Contract W-7405-Eng-48. 


\title{
The DARHT Scattering Wire Spectrometer: Operation and Checkout on ETA II
}

\author{
TJ Fessenden, consultant \\ June 14, 2005
}

\begin{abstract}
The DARHT Scattering wire energy spectrometer has been realized and checked out on ETA II. The ETA II beam energy is generally around $5.3 \mathrm{MeV}$. This value varies from pulse-topulse by around $0.5 \%$ and from month-to-month by as much as $6 \%$. The energy acceptance of the spectrometer is $\pm 5 \%$ and the time response is less than $10 \mathrm{~ns}$. The instrument was calibrated to enable absolute measurements of the ETA II beam energy accurate to $\pm 3 \%$. The beam energy in $\mathrm{MeV}$ is related to the bending magnetic field $\mathrm{B}_{\mathrm{kG}}$ according to $E_{\mathrm{MeV}}=0.511\left[\sqrt{1+347.2 B_{\mathrm{kG}}^{2}}-1\right]$.

The major difficulty encountered was in the development of detectors for the scattered electrons passing through the instrument. Fortunately one detector was fabricated that worked satisfactorily which enabled us to complete the tests on ETA II. The ETA II experiments and initial FXR experiments suggest that spurious X-ray signals will not prove troublesome. No results are yet available in the X-ray environment of DARHT.
\end{abstract}

\section{Introduction}

This paper reports progress on developing a minimally intrusive energy analyzer for use on DARHT and/or FXR. The specifications of the analyzer are:

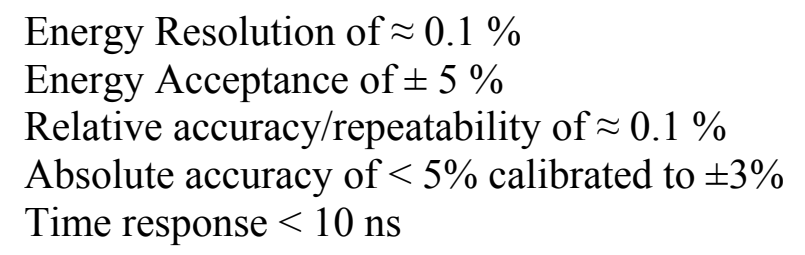

This scattering wire spectrometer was first described in a paper ${ }^{1}$ presented at the 2003 PAC conference by Art Paul. The paper focused on the concept and the optics design of the instrument. We present here a more complete description of the instrument, progress in its development, and results of energy measurements of the ETA II beam at Livermore. We conclude with a discussion of the operation and limitations of the instrument.

This energy analyzer is very similar to one ${ }^{2}$ developed for the Astron Accelerator more than 30 years ago. The principal difference is that the spectrometer is able to determine beam energies that vary as much as $\pm 5 \%$ from a mean energy. This innovation was developed to enable accurate measurements within a single pulse of beams within a $10 \%$ energy window. This requirement was driven by the very low repetition rate of the DARHT accelerator.

\section{Concept}

A sketch of the scattering wire energy analyzer is presented in Fig. 1. The instrument uses a small rod or wire placed transverse to the beam to scatter a tiny fraction of the primary 
beam into a secondary beam line. The secondary line is 11 degrees from the main line. The scattered beam travels one meter through an adjustable aperture located in front of an analyzing magnet that bends the scattered beam 60 degrees further from the main beam and focuses it to approximately a $1 \mathrm{~mm}$ wide strip at the plane of a PIN detector array $\approx 34 \mathrm{~cm}$ away. That is the image of the scattering wire at the detector plane is approximately a 4 x $10 \mathrm{~mm}$ ellipse.

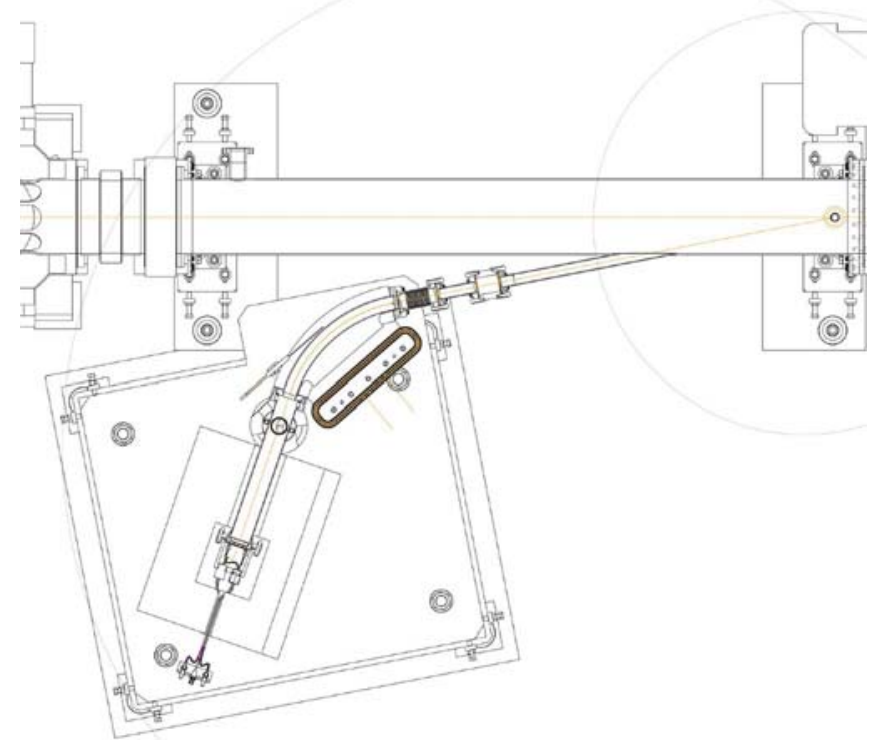

Fig. 1

An overhead sketch of the wire analyzer mounted on the accelerator vacuum system. The primary beam travels from right-to-left.

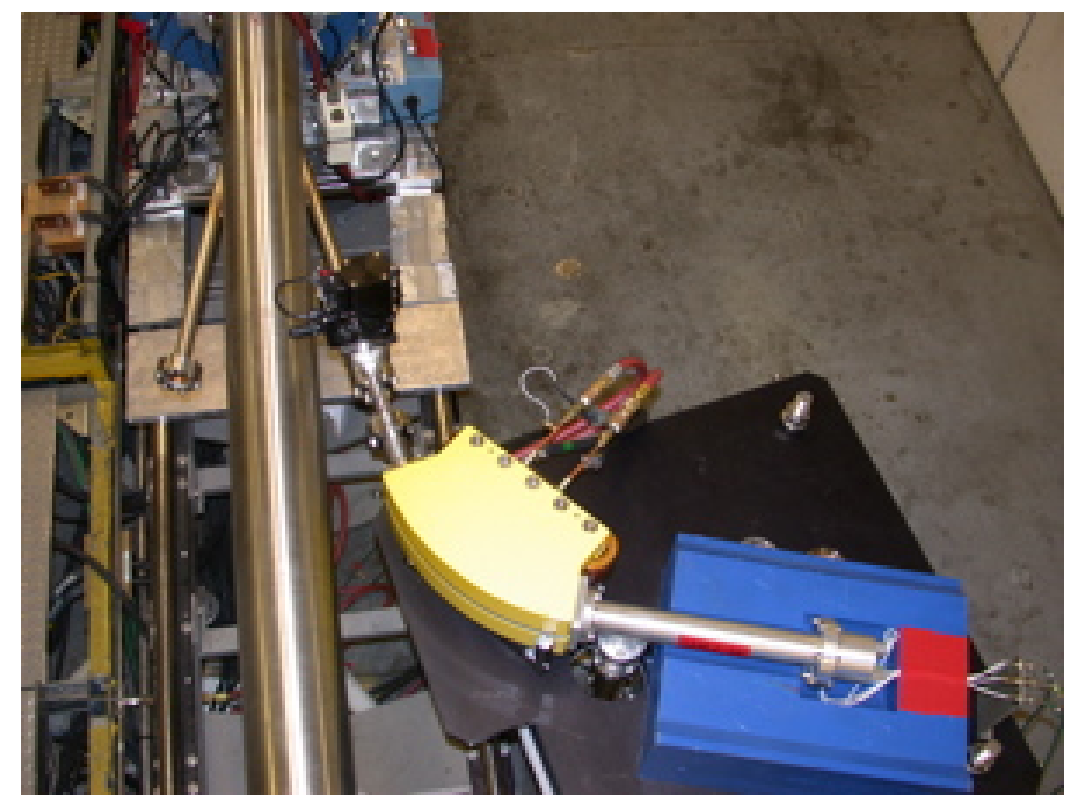

Fig. 2. Photograph of the energy analyzer mounted on ETA II The detector is at the end of the secondary vacuum line and is shielded from spurious x-rays by a blue lead box. The top half of the box was removed to facilitate the picture. 
The detector array is $4 \mathrm{~cm}$ long in the bend plane and is located transverse to the scattered beam. The energy acceptance of this detector array intercepts all electrons within $\pm 5 \%$ of the central ray energy and amplifies the scattered beam current by a factor greater then 10,000. Calculations suggest that the energy of the scattered beam can be determined with a resolution of approximately $0.1 \%$ by electrically determining the transverse position the secondary beam strikes the detector array.

\section{Magnet}

The bend magnet is very similar to the magnet ${ }^{3}$ designed by Stan Humphries for the DARHT project at Los Alamos. The coil and pole piece are the same but the gap was increased from 1 to $1.2 \mathrm{~cm}$ to permit the insertion of a separate aluminum vacuum chamber with inside dimensions of $1 \mathrm{~cm}$ high by $2 \mathrm{~cm}$ wide. The magnet requires approximately 40 Amps at ETA parameters and about 150 Amps at DARHT parameters. The field in the magnet gap is measured within an accuracy of $\pm 0.1 \%$ by a model 450 Gaussmeter manufactured by Lakeshore Cryotronics. This Gaussmeter has an IEEE-488 interface to enable remote computer monitoring.

\section{Alignment}

The curved vacuum chamber that carries the secondary is precisely secured to the magnet by pins. The magnet is aligned to the wire by adjusting the normal to the front surface of this chamber at the position of the central ray to pass through the scattering wire. In practice a small laser beam placed parallel to the central ray and perpendicular to the front chamber surface is adjusted to strike the scattering wire. The criticality of the adjustment is mitigated by the fact that the analyzer beam optics are de-magnifying by a factor of about 3 . That is, a misalignment of $1 \mathrm{~mm}$ at the wire generates a misalignment of about $1 / 3 \mathrm{~mm}$ at the detector or an error of less than $1 \%$ in energy.

\section{Scattering wire}

The scattering wire must survive the DARHT beam as well as scatter sufficient current into the analyzer magnet. The relatively long pulse width and large current density of the DARHT beam will melt most materials in a single pulse. The high melting point and relatively low density of carbon make it the best material able to survive this beam. Consequently, a carbon wire or rod was chosen as the scattering source. Although the wire intercepts less than $0.1 \%$ of the DARHT beam, it modifies the self-fields of the primary beam, which causes unacceptable modification of the beam downstream of the wire. To avoid perturbing the primary beam, a mechanism was provided that withdraws the wire without breaking vacuum when the analyzer is not in use.

At the DARHT beam energy, obtaining adequate signal at the detector requires a carbon rod or wire approximately $1 \mathrm{~mm}$ in diameter. The detector amplifies this current by a factor of 25 to 50 thousand. Fig 3 presents calculations of the anticipated current density at the entrance to the analyzing magnet as a function of wire diameter at $18 \mathrm{MeV}$ and at $5 \mathrm{MeV}$ typical of ETA II. An aperture at this location is used to control the detector signal level. For example at typical DARHT energies, a $1 \mathrm{~mm}$ scattering wire; a $3 \mathrm{~mm}$ round aperture; and a chip gain of 25,000 should produce a signal level of about 5 Volts into $50 \mathrm{Ohms}$. At ETA II energies a $0.25 \mathrm{~mm}(10$ mil) scattering wire will produce the same 5 Volt signal level. 



Fig 3 Estimates of the signal current density at the entrance to the analyzing magnet at 18.3 and $5 \mathrm{MeV}$

The energy lost in the scattering wire is not negligible and can amount to as much as 1 or $2 \%$. Fortunately, calculations using the MCNP Monte Carlo particle code ${ }^{4}$ developed at LANL indicate that the energy spectrum is spread by a comparable amount and the peak is representative of the beam energy. Fig 4 shows Jim McCarrick's calculation of the spectrum at the detector produced by a monoenergetic $5 \mathrm{MeV}$ beam striking the 20 mil $(0.5 \mathrm{~mm})$ carbon wire used in the ETA II experiments. The peak is shifted downward by more than $100 \mathrm{kV}$ or $2 \%$, and the half-width of the spread spectrum is approximately $50 \mathrm{kV}$ or $1 \%$. This is $1 / 10^{\text {th }}$ the effective energy acceptance of the detector.

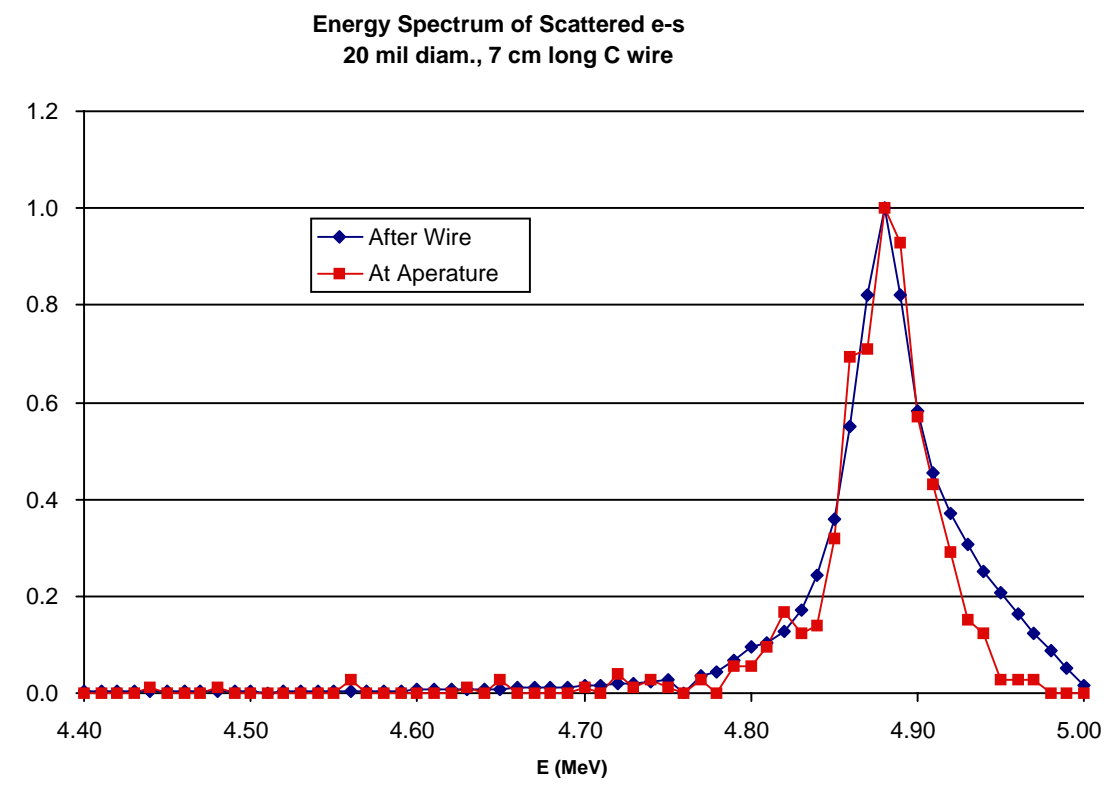

Fig. 4. Jim McCarrick's MCNP code calculation of the electron energy spectrum of a $5 \mathrm{MeV}$ electron beam scattered by a 20 mil carbon wire. 


\section{Signal Strength}

The point design tended to maximize the amplitude of the detector signal under the philosophy that too much signal is better than too little. Calculations suggest that signal voltages could be several hundred volts, which are much more than adequate, and more than the PIN detector can provide. The aperture in front of the magnet can be varied and was included to provide a simple way of reducing the signal if required. On ETA II we found that a 1/8 inch aperture was optimum. Using a smaller scattering wire would also be very effective in lowering signal strength. Notice that these strategies would also improve the resolution of the instrument.

\section{Detector}

The electrons passing through the analyzing magnet are detected using a PIN detector placed $30 \mathrm{~cm}$ from the magnet exit at the focus of the system. A sketch of the detector showing the beam image (red ellipse) on the detector and its associated electrical circuit is shown in Fig. 5. It consists of a rectangular chip approximately $2.5 \mathrm{~mm}$ wide, $0.25 \mathrm{~mm}$ thick by $40 \mathrm{~mm}$ long placed perpendicular to the central ray and orientated so that the long dimension is in the bend plane of the magnet. The detector is placed at the optical focus of the secondary beam system. That is, the scattering wire is imaged at the detector center by the focusing action of the magnet. The system provided essentially no focusing in the direction perpendicular to the bend plane of the magnet. As the mean beam energy varies during the accelerator pulse, the image of the wire moves along the detector chip. The $40 \mathrm{~mm}$ detector subtends an angle corresponding to a total energy sweep of about $10 \%$ (actually $9.8 \%$ ) or $\pm 5 \%$ from the center.

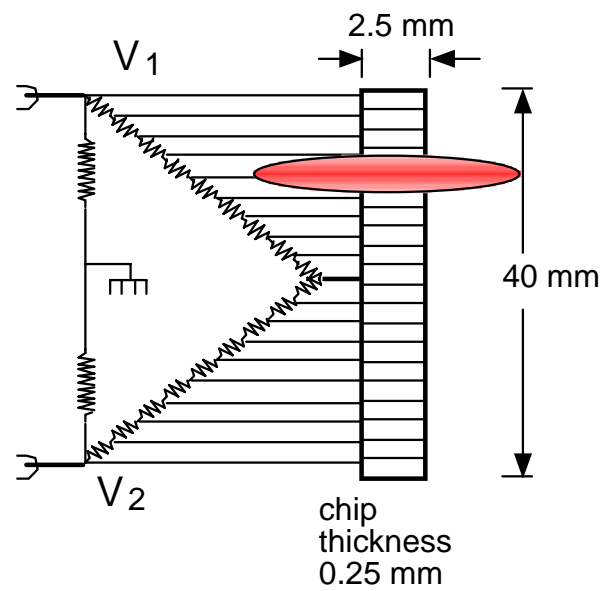

Fig. 5 Sketch of the PIN detector showing the secondary beam image striking the detector and a part of the bridge circuit that permits detection of the position of the beam centroid on the chip

The detector was divided into 40 segments as shown in the sketch. Each of these was connected to a bridge circuit that was used to determine the location on the chip struck by the beam. During operation, the diode chip is back biased (not shown in sketch) to about 250 volts. The individual resisters in the bridge circuit are each $2.49 \mathrm{Ohms}$. The two outputs feed the voltages $\mathrm{V}_{1}$ and $\mathrm{V}_{2}$ though terminated $50 \mathrm{Ohm}$ cables to an oscilloscope. Two $10 \mathrm{~K}$ resistors that protect the chip if the cables are not terminated are also shown. The beam position on the chip is then given by: 


$$
x / 40=\% / 10=0.5 \frac{V_{1}-V_{2}}{V_{1}+V_{2}}
$$

Here $\mathrm{x}$ is the distance from center measured in $\mathrm{mm}$. However, the most useful relation is the second where $\%$ is the percent the beam is away from the central ray energy. Brett Raymond has incorporated this equation along with the calibration equation, Eq. 3, into the SUICIDE suite of data processing codes.

The focal plane is inclined about 38 degrees to the detector chip and as a consequence only the beam image at chip center is in perfect focus. However, the largest spread in the beam image results from the energy spread generated by the scattering wire. Fig. 4 shows that the 20mil wire used in the experiment spread the beam image about $1 \%$ in the energy plane. This is $1 / 10$ th the energy range of the detector. A much more complete discussion of the beam optics is presented in reference 1 .

The detector is back-biased to a voltage of a few hundred volts. Energetic electrons from the detected beam generate electron hole-pairs for each 3.7 Volts lost in passing through the detector. The bias field then sweeps the hole-pairs out. Since each energetic electron will loose about 100,000 Volts in passing through the detector, the detector current gain will be on the order of 25,000. A bias voltage of 100 to 200 volts is required to sweep the electron-hole pairs from the $0.25 \mathrm{~mm}$ thick detector in $10 \mathrm{~ns}$ or less. Bob Kuckuck has provided an excellent discussion of the properties of PIN diodes for detecting energetic electrons in Ref. 5.

The detector was fabricated using the resources of the laboratory. We encountered several problems with the processes involved in the PIN chip fabrication, and continue to have issues with excessive leakage current that leads to thermal runaway. Fortunately, one satisfactory detector was fabricated which enabled the checkout of the instrument on ETA-II. However, it was necessary to pulse-bias the detector to prevent thermal runaway. The bias voltage was applied about $1 \mathrm{~ms}$ before the ETA II pulse and removed about $4 \mathrm{~ms}$ after the pulse.

Bill Nexsen has suggested an alternate equivalent configuration for the detector. It consists of a single rectangular PIN detector bisected by a cut connecting two opposite corners. This detector would require only two connections instead of 40 to the face and the common connection to the back. This could possibly simplify the fabrication of the chip. A potential down side is that the full signal voltage, which can be as large as 100 Volts, will appear between the two halves of the chip and across the cut. However, this approach could ultimately prove more successful and should be tried.

\section{Shielding}

The PIN detector is also sensitive to x-rays and shielding is required. A TLD survey of the radiation generated by the DARHT I accelerator reported by Alfred Cucchiara in May 2002 revealed x-ray intensities from $13 \mathrm{mR}$ per pulse directly behind the beam stop to $1500 \mathrm{mR} / \mathrm{pulse}$ directly in front of the beam stop. Radiation 14.4 inches to either side of the beam stop amounted to approximately $325 \mathrm{mR} /$ pulse. Glen Westenskow used the following arguments to estimate the shielding requirement to mitigate $x$-ray induced noise. Since the DARHT II pulse is 
20 times longer than the DARHT I pulse, we should expect radiation at the position of the PIN detector to be somewhere near $10 \mathrm{R} /$ pulse. The mass of the detector is $.06 \mathrm{~g}$ $(4 \mathrm{~cm})(0.25 \mathrm{~cm})(.025 \mathrm{~cm})\left(2.32 \mathrm{~g} / \mathrm{cm}^{3}\right)$. If unshielded, this radiation would deposit $6 \mu \mathrm{J} / \mathrm{pulse}$ in the detector. $\left(1 \mathrm{R}=10^{-5} \mathrm{~J} / \mathrm{g}\right)$. Glen estimates that four inches of lead shielding will attenuate this by a factor of 100 . At a detector current of $10 \mu \mathrm{A}$, the electrons traversing the detector will deposit approximately $2.3 \mu \mathrm{J} / \mathrm{pulse}$. Thus the signal/noise ratio in the detector should be about 40, which would be quite acceptable.

\section{Energy Calibration}

The relation between the energy of the detected beam and the magnetic bending field in the magnet is given by

$$
E_{M e V}=E_{\text {rest }}\left[\sqrt{1+\left(r_{c m} B_{k G} / 1.705\right)^{2}}-1\right]
$$

Here $\mathrm{E}_{\mathrm{MeV}}$ is the energy of the detected beam along the central ray of the instrument, $\mathrm{E}_{\text {rest }}$ is the rest energy of an electron $(0.511 \mathrm{MeV}), \mathrm{r}_{\mathrm{cm}}$ is the effective radius of curvature of the central ray in the magnet, and $\mathrm{B}_{\mathrm{kG}}$ is the bending magnetic field at the central ray in kilogauss. (1.705 is $\mathrm{mc} / \mathrm{e}$ in the proper units). For an idealized bend field (uniform with no fringe fields) $r_{\mathrm{cm}}$ equals the physical radius of curvature of the central ray through the magnet.

The effective radius of curvature $r_{\mathrm{cm}}$ of the DARHT spectrometer* ${ }^{*}$ was determined experimentally ${ }^{6}$ at the Bechtel Los Alamos calibration facility to be 31.77 centimeters with an accuracy of $\pm 3 \%$ over the range 2.5 to $20 \mathrm{MeV}$. The radius of curvature of central ray in the magnet shown in Fig. 1 is $29.845 \mathrm{~cm}$. This is about $6.5 \%$ smaller than the effective radius of curvature $r_{\mathrm{cm}}$ of the detected beam principally due to the fringe fields that exist at the magnet entrance and exit. Using the experimental value the beam energy is given to about $\pm 3 \%$ accuracy by

$$
E_{\mathrm{MeV}}=0.511\left[\sqrt{1+347.2 B_{k G}^{2}}-1\right] \quad(\text { accuracy of } \pm 3 \%)
$$

The Bechtel calibration facility uses negative ion beams of several species including hydrogen and oxygen at energies of 10 to $30 \mathrm{KeV}$ to exactly anticipate the trajectories of electrons at energies of a few to over $20 \mathrm{MeV}$ through the spectrometer. The bending and/or optics of beams passing through magnetic systems depend only on the charge and momentum of the particle. Electrons or negative ions of single charge state having identical momenta travel through the spectrometer on identical trajectories. A d.c. acceleration potential that is known to an accuracy of $0.1 \%$ produces the ion beams. Thus the negative ion beams should in principal be able to provide instrument calibrations in the range of interest for DARHT, ETA, and FXR to an accuracy approaching $0.1 \%$. Lack of reproducibility appears at present to limit the accuracy to something like $\pm 2 \%$.

\footnotetext{
${ }^{*}$ A second spectrometer was calibrated at Bechtel Los Alamos ${ }^{7}$ and is presently deployed on FXR. The calibration for this instrument is $E_{M e V}=0.511\left[\sqrt{1+374.75 B_{k G}^{2}}-1\right]$ (accuracy of $\pm 2 \%$ ). The total energy acceptance of the $4 \mathrm{~cm}$ detector chip is $9.1 \%$.
} 
At Livermore we have been simply setting the magnet field at the proper value to detect the beam and counting on the fact that we measure the field directly to eliminate the effects of hysteresis. Experiments at Los Alamos showed that this procedure yielded results at ETA II parameters that were repeatable to only $\pm 1 \%$. A better procedure, used at Los Alamos, is to first cycle the magnet from negative to positive saturation then down to the proper current to pass the beam through the spectrometer. This procedure improves the repeatability to $\pm 0.3 \%$ or better. This result suggests that the bend-field varies in shape as well as amplitude at different parts of the hysteresis curve. Experiments indicated that this variation is most significant at the entrance and exit of the magnet: amounting to near one percent at these points. Even though most of the magnet iron is far from saturation, the flux is strongly concentrated at the sharp edges at the magnet entrances and exits resulting in to areas of saturation at these points. Moreover, probing with a small permanent magnet revealed that the stainless steel flange that was explosively bonded to the aluminum vacuum chamber and some of the stainless steel screws at the bendmagnet entrance and output flanges had become slightly magnetic. These phenomena are likely to be more important at the higher DARHT II and FXR energies suggesting that the Los Alamos procedure should be used.

\section{Energy Dispersion at the Detector}

Art Paul calculated the energy dispersion at the plane of the detector as $2.32 \% / \mathrm{cm}$. This result is contained in Fig 3 of reference 1 (although it may not be readable). The energy dispersion was also measured by the Bechtel Los Alamos team using a five slotted mask in front of their detector to be $2.8 \% / \mathrm{cm}$. Using Art's number we find the total energy acceptance of the 4 $\mathrm{cm}$ detector to be $9.3 \%$ while the Bechtel results would suggest $11.1 \%$.

\section{Experimental Results from ETA II}

Program priorities permitted only a few measurements of the ETA II beam energy to be made using this instrument. The first was on November 17, 2003 using the one detector that was successfully fabricated. In all of these experiments a $0.5 \mathrm{~mm}$ (20 mil) carbon rod was used as the scattering wire in all of these experiments. As a consequence the image on the detector subtended about 1/10 the detector length or an "energy" of $0.05 \mathrm{MeV}$. Due to energy loss in the carbon scattering wire, the energies of all the data presented in Figs. 6, 7, and 9 should be increased by approximately $0.11 \mathrm{MeV}$.

The data contained in Figs. 6 through 9 were processed by an energy spectrometer extension written by Brett Raymond to the SUICIDE data analysis suite. This extension incorporated the detector relation of Eq. 1 and avoided the division by small numbers when the signal sum voltage was small. Much more data were taken in each case. For clarity, only those data from measurements near the mean ETA II energy are presented. 


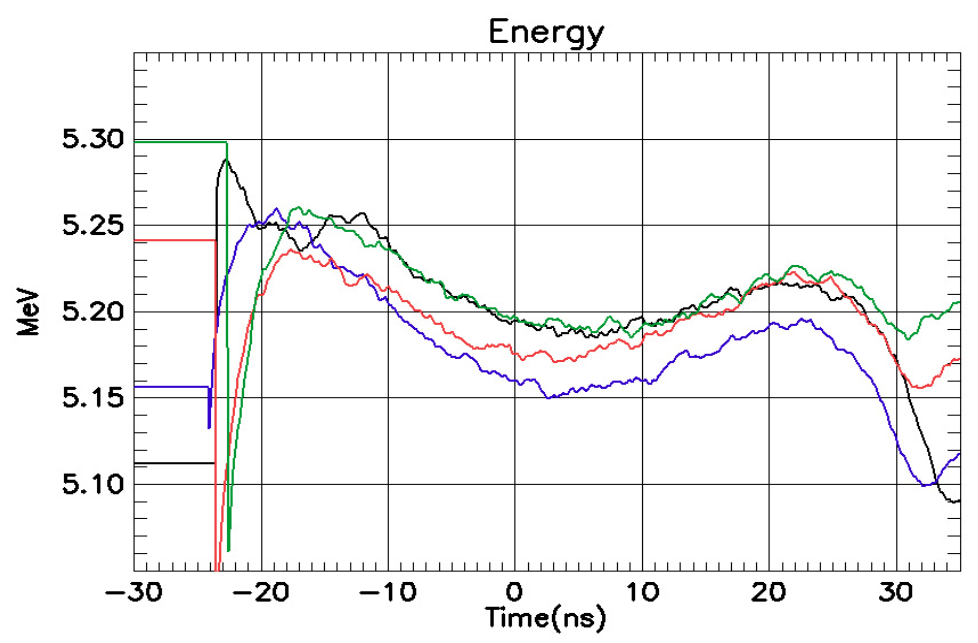

Fig 6. Measurements of the ETA II beam energy versus time on November 17, 2003. The central ray energy was set to the four different values shown at the abscissa by small changes in the strength of the bending magnetic field. Due to energy loss in the scattering wire these data are approximately $0.11 \mathrm{MeV}$ too low.

The data shown are for four similar pulses of the ETA II accelerator. For these, the central ray energy of the analyzer was varied from 5.11 MeV (black curve) to $5.30 \mathrm{MeV}$ (green curve). This figure illustrates the action of the position determining circuit described in the Detector description section of the report. In the ideal situation these traces would exactly overlay.

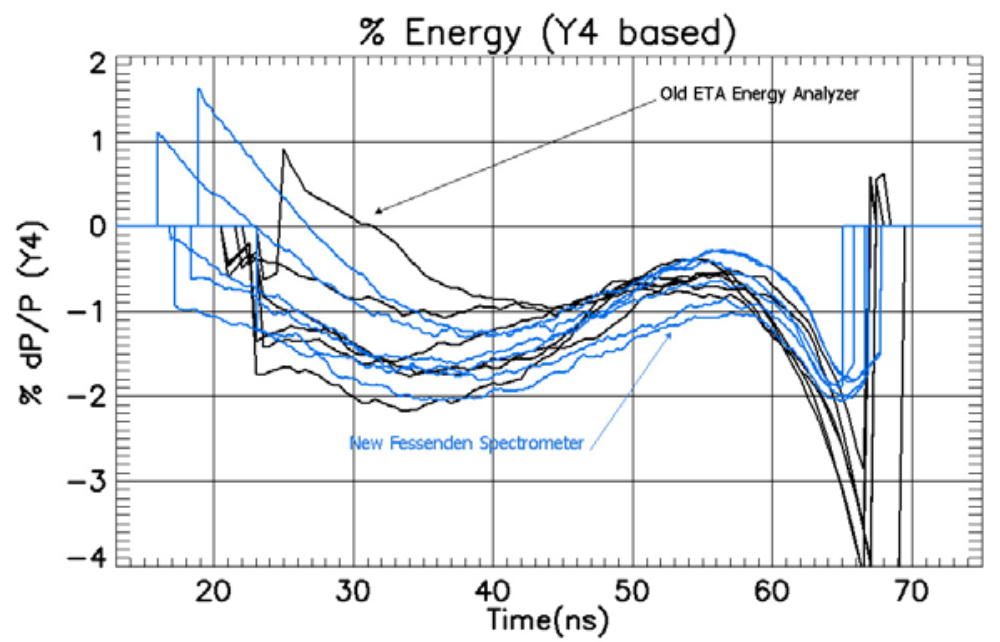

Fig 7. Comparisons of relative measurements of the ETA II beam energy using the old energy analyzer and the wire spectrometer on November 17, 2003. The fields of the two analyzers were held constant for these measurements.

Relative measurements of the ETA II beam obtained with the wire analyzer were compared with those from the old energy analyzer and are presented in Fig. 6. These data suggest that the lack of repeatability of both the wire spectrometer and the old energy analyzer is most likely due to pulse-to-pulse variations of the ETA accelerator. This variation is approximately $0.5 \%$. 


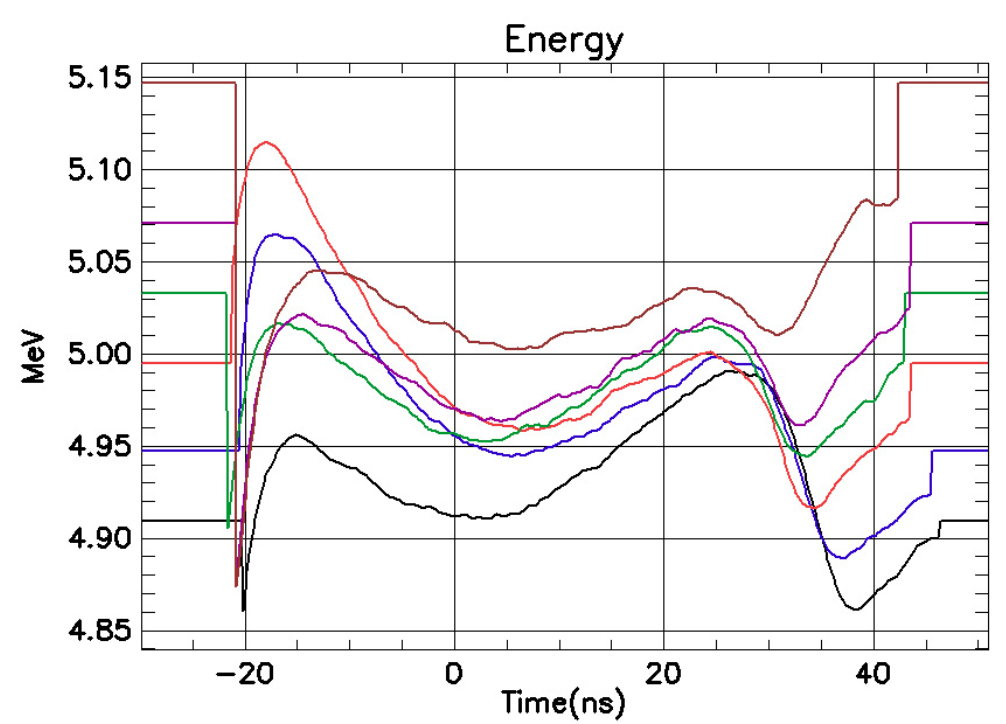

Fig. 8 Measurement of the ETA II beam energy performed on January 14, 2004. At that time the ETA II accelerator appeared to be operating at just over $5 \mathrm{MeV}$ (after correction for the energy loss in the wire).

A second measurement of ETA II energy was obtained on January 14, 2004. At that time the beam energy was some $300 \mathrm{KeV}$ lower than normal. These data are presented in Fig. 8. Here the central ray energy was varied from $4.91 \mathrm{MeV}$ (black curve) to nearly $5.15 \mathrm{MeV}$ (brown curve).

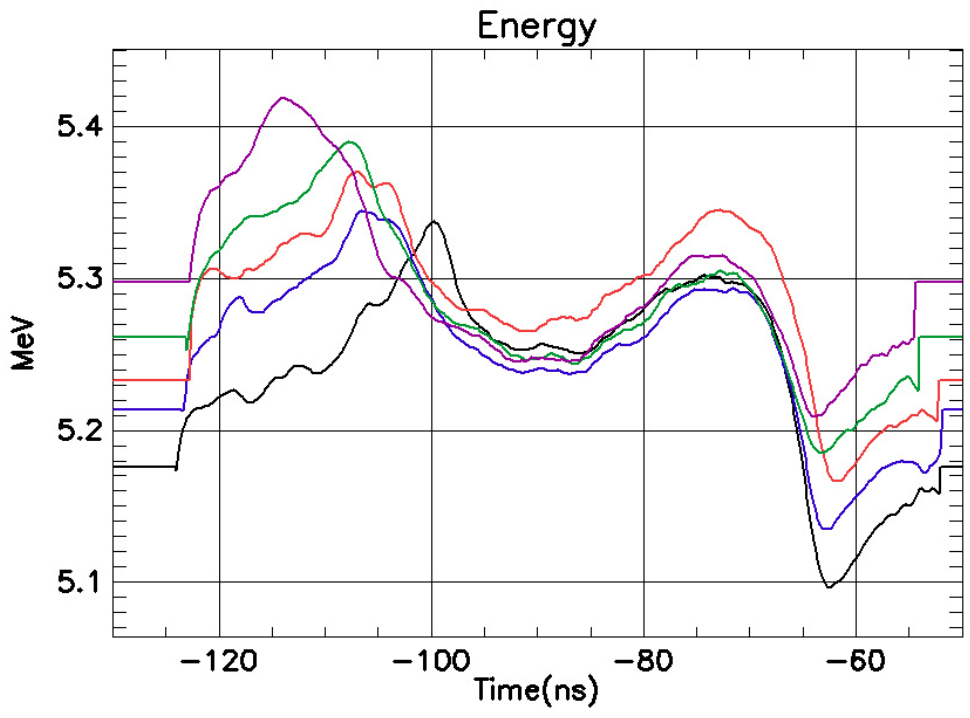

Fig. 9. The ETA II beam energy on July 13, 2004. A larger than normal energy spike on the front of the pulse is responsible for the evolution of these traces.

The last measurement of the ETA II beam shown in Fig. 9 was obtained on July 13, 2004. At that time the accelerator was operating with central pulse energy near $5.4 \mathrm{MeV}$ but there was a relatively large energy spike near the front of the pulse. As a result the wire image at the beginning of the pulse tended to fall off the edge of the detector chip; particularly so when the central ray energy was adjusted to lower energies. Again the central ray energy was varied from $5.18 \mathrm{MeV}$ (black curve) to $5.30 \mathrm{MeV}$ (violet curve). 


\section{Conclusions and Comments}

The instrument works as designed with even some improvement over the original specifications. The energy acceptance of $\pm 5 \%$ is twice the original specification. With the addition of the calibration provided by the team at Bechtel Los Alamos, absolute energy measurements with accuracy of $\pm 3 \%$ or better are also possible. The repeatability and accuracy could be improved by using the Los Alamos magnet adjustment procedure. The major remaining difficulty is the development of satisfactory detectors for the secondary beam. We are operating with the one detector that was successfully fabricated. Until more detectors are available, operation is tenuous at best. Steve Falabella has ordered PIN detectors from an offsite source. These have been received and are awaiting testing and evaluation on ETA II.

The scattering wire must be carefully matched to the accelerator energy to minimize the spread in the secondary beam image at the detector. The 20-mil carbon wire was at least twice as thick as optimum for use with ETA II. Unfortunately, smaller diameter carbon wires were not available at the time of these experiments.

Contrary to the experience on ETA II, the instrument should be fully installed at its next deployment. This includes hooking up the special vacuum system that is a part of the instrument and integrating the digital output of the Gaussmeter with the data analysis system. Much time and confusion would have been saved had we made the effort to properly install the instrument.

The operation of the instrument in the x-ray environments of DARHT and FXR will require careful consideration of the stopping point of the beam with respect to the location of the detector. If the detector falls into or near the forward x-ray cone at the beam stop, it is unlikely that the detector shielding will be adequate. Experiments on ETA-II showed that, even with the shielding, an unacceptably large x-ray noise signal was present if the beam was spilled near the detector. A further problem resulting from energetic electrons scattering off the vacuum wall into the acceptance cone of the analyzer was also apparent.

The analyzer alignment procedure should have received more consideration. The method developed by our colleagues at Los Alamos is far superior. A window in the curved vacuum section within the analyzing magnet that affords a view through the input aperture and back toward the wire should have been provided. This would have permitted a much simpler and more accurate method of aligning the analyzer to the wire. I accept responsibility for this omission.

\section{Acknowledgement}

Many people contributed to the realization of this instrument over the past 3 years. Most notably at LLNL: Shmuel Eylon, Steve Falabella, Steve Hawkins, Bryan Lee, Jim MacCarrick, Art Paul, Brett Raymond, Jim Sullivan, Jim Watson, John Weir, and Glen Westenskow. At Los Alamos: Dick Bartsch, Angela Tipton, and Rusty Trainham. I enjoyed working with each of these people and thank them for their contributions. 


\section{References}

1) A.C. Paul et al, "The DARHT II Energy Analyzer", Presented at the PAC conference, Portland, Oregon, May 12-16, 2003.

2) T.J. Fessenden, “The Astron On-Line Beam Energy Analyzer,’Rev. of Sci. Instru., 43, 1090, (1972)

3) S. Humphries Jr., "Magnetic Field Calculations, 60 Degree DARHT Spectrometer", Acceleration Associates, (April 1994).

4) Briesmeister, Judith, Editor MCNP - A General Monte Carlo N-Particle Transport Code, Version 4a. LA - 12525 Manual: Los Alamos National Laboratory; 1988.

5) R.W. Kuckuck, "Semiconductor Detectors for use in the Current Mode", UCRL 51011, Feb 10, 1971.

6) The effective magnet bend radius was deduced from the calibration provided by A. Tipton in her E-Mail Communication of Feb. 3, 2005.

7) A. Tipton, E-Mail Communication of June 6, 2005. 